\title{
Various Empirical Equations for the Electromagnetic Force in Terms of the Cosmic Microwave Background Temperature
}

\author{
Tomofumi Miyashita \\ Miyashita Clinic, Osaka, Japan \\ Email: tom_miya@plala.or.jp
}

How to cite this paper: Miyashita, T. (2021) Various Empirical Equations for the Electromagnetic Force in Terms of the Cosmic Microwave Background Temperature. Journal of Modern Physics, 12, 623-634.

https://doi.org/10.4236/jmp.2021.125040

Received: February 13, 2021

Accepted: April 18, 2021

Published: April 21, 2021

Copyright $\odot 2021$ by author(s) and Scientific Research Publishing Inc. This work is licensed under the Creative Commons Attribution International License (CC BY 4.0).

http://creativecommons.org/licenses/by/4.0/

\begin{abstract}
Previously, we proposed an empirical equation describing the relationship between the gravitational force and the temperature of the cosmic microwave background (CMB). After evaluating our equation, we discovered many empirical equations describing the electromagnetic force in terms of the $\mathrm{CMB}$, including equations for the Rydberg constant, the Bohr radius, the Compton wavelength, the classical electron radius, the Hartree energy, the Coulomb's law with distance, and the ratio between the gravitational force and electric force. The background theory is not yet complete. However, we can justify why the discovered empirical equations should not be coincidence.
\end{abstract}

\section{Keywords}

Temperature of the Cosmic Microwave Background

\section{Introduction}

Previously, we reported the following equation [1] [2]:

$$
\frac{G m_{p}}{\frac{\lambda_{p}}{2}} \times 1 \mathrm{~kg}=\frac{9}{2} k T_{c}
$$

where $G, m_{p}, \lambda_{p}, k, T_{c}$ and $1 \mathrm{~kg}$ are the gravitational constant, the rest mass of a proton, the Compton wavelength, the Boltzmann constant, the temperature of the cosmic microwave background (CMB) and the standard unit of mass, respectively. Then,

$$
\frac{G m_{p}}{\frac{\lambda_{p}}{2}} \times 1 \mathrm{~kg}=\frac{6.6743 \times 10^{-11} \times 1.6726 \times 10^{-27}}{\frac{1.3241 \times 10^{-15}}{2}}=1.6897 \times 10^{-22}(\mathrm{~J})
$$




$$
\begin{gathered}
\frac{9}{2} k T_{c}=\frac{9}{2} \times 1.3807 \times 10^{-23} \times 2.7255=1.6933 \times 10^{-22}(\mathrm{~J}) \\
\text { Error }=\frac{1.6897 \times 10^{-22}}{1.6933 \times 10^{-22}}-1=-0.00217
\end{gathered}
$$

The temperature $\left(T_{c}\right)$ calculated from Equation (1) is $2.71957 \mathrm{~K}$, and the measured CMB is $2.72548 \mathrm{~K}$. Accordingly, using Jarzynski's equality [3] and Ted Jacobson's theory [4], we attempted to explain Equation (1).

According to Jarzynski, Jarzynski's equality may be useful for quantum mechanics [3]. We searched for empirical equations for the electromagnetic force in terms of the temperature of the cosmic microwave background using Excel sheets. This search method relied almost entirely on chance, requiring much time and perseverance. In this way, we discovered several empirical equations. Unfortunately, the background theory still could not be sufficiently completed. Therefore, we abandoned the attempt to provide a theoretical explanation in this report. However, we can justify why the discovered empirical equations should not be coincidence.

The rest of the paper is organized as follows. In Section 2, we present the symbol list and the calculation results for frequently used values. In Section 3, we present our empirical equations. In Section 4, we explain the relationships among our empirical equations.

\section{Symbol List and Frequently Used Values}

\subsection{Symbol List}

These values were obtained from Wikipedia.

G: gravitational constant: $6.6743 \times 10^{-11}\left(\mathrm{~m}^{3} \cdot \mathrm{kg}^{-1} \cdot \mathrm{s}^{-2}\right)$

$T_{\dot{c}}$ temperature of the cosmic microwave background: $2.72548(\mathrm{~K})$

$k$. Boltzmann constant: $1.380649 \times 10^{-23}\left(\mathrm{~J} \cdot \mathrm{K}^{-1}\right)$

c. speed of light: $299792458(\mathrm{~m} / \mathrm{s})$

$h$ : Planck constant: $6.62607015 \times 10^{-34}$ (Js)

$\hbar$ : Dirac constant (reduced Planck constant): $1.054571817 \times 10^{-34}$ (Js)

$\mathcal{E}_{0}$ : electric constant: $8.8541878128 \times 10^{-12}\left(\mathrm{~N} \cdot \mathrm{m}^{2} \cdot \mathrm{C}^{-2}\right)$

$\mu_{0}$ : magnetic constant: $1.25663706212 \times 10^{-6}\left(\mathrm{~N} \mathrm{~A}^{-2}\right)$

$e$ electric charge of one electron: $-1.602176634 \times 10^{-19}(\mathrm{C})$

$q_{m}$ : magnetic charge of one magnetic monopole: $4.13566770 \times 10^{-15}(\mathrm{~Wb})$

(this value is only a theoretical value, $q_{m}=h / e$ )

$m_{p}$ : rest mass of a proton: $1.672621923 \times 10^{-27}(\mathrm{~kg})$

$m_{e}:$ rest mass of an electron: $9.1093837 \times 10^{-31}(\mathrm{~kg})$

$\lambda_{p}$ : Compton wavelength for a proton: $1.32141 \times 10^{-15}(\mathrm{~m})$

$\lambda_{e}$ : Compton wavelength for an electron: $2.4263102367 \times 10^{-12}(\mathrm{~m})$

$r_{e}$ : classical electron radius: $2.8179403227 \times 10^{-15}(\mathrm{~m})$

$a_{0}$ : Bohr radius: $0.529177210 \times 10^{-10}(\mathrm{~m})$

$R_{\infty}$ : Rydberg constant: $10973731.568\left(\mathrm{~m}^{-1}\right)$ 
$E_{h}$ : Hartree energy: 27.211386245988 (eV)

$R_{k}$ : von Klitzing constant: $25812.80745(\Omega)$

$Z_{0}$ : wave impedance in free space: $376.730313668(\Omega)$

$\alpha$ : fine-structure constant: 1/137.0359991

\subsection{Calculation Results for Frequently Used Values}

The calculation results for several frequently used values are presented in this section. The number of significant figures used is 5 .

$$
\begin{gathered}
\frac{e^{2}}{4 \pi \varepsilon_{0}}=\frac{\left(1.6022 \times 10^{-19}\right)^{2}}{4 \pi \times 8.8542 \times 10^{-12}}=2.3071 \times 10^{-28}(\mathrm{~J} \cdot \mathrm{m}) \\
\frac{e}{4 \pi \varepsilon_{0}}=\frac{1.6022 \times 10^{-19}}{4 \pi \times 8.8542 \times 10^{-12}}=1.4400 \times 10^{-9}(\mathrm{~J} \cdot \mathrm{m} / \mathrm{C}) \\
\frac{e^{2}}{4 \pi \varepsilon_{0}} \frac{e}{4 \pi \varepsilon_{0}}=2.3071 \times 10^{-28} \times 1.4400 \times 10^{-9}=3.3221 \times 10^{-37}\left(\mathrm{~J}^{2} \cdot \mathrm{m}^{2} \cdot \mathrm{C}^{-1}\right) \\
k T_{c}=1.3807 \times 10^{-23} \times 2.7255=3.7629 \times 10^{-23}(\mathrm{~J})
\end{gathered}
$$

\section{Our Empirical Equations}

We present our empirical equations and their verification and errors in detail.

\subsection{Four Special Lengths}

First, we present the empirical equations for four special lengths.

\subsubsection{Classical Electron Radius}

$$
\frac{1}{\frac{2 r_{e} \pi}{2}} \times \frac{e^{2}}{4 \pi \varepsilon_{0}} \times \frac{e}{4 \pi \varepsilon_{0}} \times\left(1 \frac{\mathrm{C}}{\mathrm{J} \cdot \mathrm{m}}\right)=k T_{c}
$$

where $1 \mathrm{C} / \mathrm{J} / \mathrm{m}$ is the standard electrostatic quantity, which is explained in a later section.

$$
\begin{gathered}
\frac{1}{\frac{2 r_{e} \pi}{2}} \frac{e^{2}}{4 \pi \varepsilon_{0}} \frac{e}{4 \pi \varepsilon_{0}} \times\left(1 \frac{\mathrm{C}}{\mathrm{J} \cdot \mathrm{m}}\right)=\frac{3.3221 \times 10^{-37}}{\pi \times 2.8179 \times 10^{-15}}=3.7526 \times 10^{-23}(\mathrm{~J}) \\
\text { Error }=\frac{3.7526 \times 10^{-23}}{3.7629 \times 10^{-23}}-1=-0.00274
\end{gathered}
$$

\subsubsection{Compton Wavelength for an Electron}

$$
\begin{gathered}
\frac{1}{\frac{\lambda_{e}}{2}} \times \frac{1}{\alpha} \times \frac{e^{2}}{4 \pi \varepsilon_{0}} \times \frac{e}{4 \pi \varepsilon_{0}} \times\left(1 \frac{\mathrm{C}}{\mathrm{J} \cdot \mathrm{m}}\right)=k T_{c} \\
\frac{1}{\frac{\lambda_{e}}{2}} \frac{1}{\alpha} \frac{e^{2}}{4 \pi \varepsilon_{0}} \frac{e}{4 \pi \varepsilon_{0}} \times\left(1 \frac{\mathrm{C}}{\mathrm{J} \cdot \mathrm{m}}\right)=\frac{3.3221 \times 10^{-37} \times 137.036}{2.4263 \times 10^{-12}}=3.7526 \times 10^{-23}(\mathrm{~J})
\end{gathered}
$$




$$
\text { Error }=\frac{3.7526 \times 10^{-23}}{3.7629 \times 10^{-23}}-1=-0.00274
$$

\subsubsection{Bohr Radius}

$$
\begin{aligned}
& \frac{1}{\frac{2 \pi a_{0}}{2}} \times \frac{1}{\alpha^{2}} \times \frac{e^{2}}{4 \pi \varepsilon_{0}} \times \frac{e}{4 \pi \varepsilon_{0}} \times\left(1 \frac{\mathrm{C}}{\mathrm{J} \cdot \mathrm{m}}\right)=k T_{c} \\
& \frac{1}{\pi a_{0}} \frac{1}{\alpha^{2}} \frac{e^{2}}{4 \pi \varepsilon_{0}} \frac{e}{4 \pi \varepsilon_{0}} \times\left(1 \frac{\mathrm{C}}{\mathrm{J} \cdot \mathrm{m}}\right) \\
& =\frac{3.3221 \times 10^{-37} \times(137.036)^{2}}{\pi \times 5.2918 \times 10^{-11}}=3.7526 \times 10^{-23}(\mathrm{~J}) \\
& \text { Error }=\frac{3.7526 \times 10^{-23}}{3.7629 \times 10^{-23}}-1=-0.00274
\end{aligned}
$$

\subsubsection{Rydberg Constant}

$$
\begin{gathered}
\frac{1}{\frac{1}{4 R_{\infty}}} \times \frac{1}{\alpha^{3}} \times \frac{e^{2}}{4 \pi \varepsilon_{0}} \times \frac{e}{4 \pi \varepsilon_{0}} \times\left(1 \frac{\mathrm{C}}{\mathrm{J} \cdot \mathrm{m}}\right)=k T_{c} \\
\frac{1}{\frac{1}{4 R_{\infty}}} \frac{1}{\alpha^{3}} \frac{e^{2}}{4 \pi \varepsilon_{0}} \frac{e}{4 \pi \varepsilon_{0}} \times\left(1 \frac{\mathrm{C}}{\mathrm{J} \cdot \mathrm{m}}\right) \\
=\frac{3.3221 \times 10^{-37} \times(137.036)^{3}}{\frac{1}{4 \times 1.0973 \times 10^{7}}}=3.7526 \times 10^{-23}(\mathrm{~J}) \\
\text { Error }=\frac{3.7526 \times 10^{-23}}{3.7629 \times 10^{-23}}-1=-0.00274
\end{gathered}
$$

\subsection{Two Special Energies}

We next present the empirical equations for two special energies.

\subsubsection{Rest Mass of an Electron}

$$
\begin{gathered}
\frac{m_{e} c^{2}}{e \pi} \times \frac{e^{2}}{4 \pi \varepsilon_{0}} \times\left(1 \frac{\mathrm{C}}{\mathrm{J} \cdot \mathrm{m}}\right)=k T_{c} \\
m_{e} c^{2}=9.1094 \times 10^{-31} \times\left(2.9979 \times 10^{8}\right)^{2}=8.1871 \times 10^{-14}(\mathrm{~J}) \\
\frac{m_{e} c^{2}}{e \pi} \frac{e^{2}}{4 \pi \varepsilon_{0}} \times\left(1 \frac{\mathrm{C}}{\mathrm{J} \cdot \mathrm{m}}\right)=\frac{8.1871 \times 10^{-14} \times 2.3071 \times 10^{-28}}{\pi \times 1.6021 \times 10^{-19}}=3.7526 \times 10^{-23}(\mathrm{~J}) \\
\text { Error }=\frac{3.7526 \times 10^{-23}}{3.7629 \times 10^{-23}}-1=-0.00274
\end{gathered}
$$

\subsubsection{Hartree Energy}

$$
E_{h} \times \frac{1}{\alpha^{2}} \times \frac{1}{e \pi} \times \frac{e^{2}}{4 \pi \varepsilon_{0}} \times\left(1 \frac{\mathrm{C}}{\mathrm{J} \cdot \mathrm{m}}\right)=k T_{c}
$$




$$
\begin{gathered}
\frac{E_{h}}{e \pi} \frac{e^{2}}{4 \pi \varepsilon_{0}} \times\left(1 \frac{\mathrm{C}}{\mathrm{J} \cdot \mathrm{m}}\right) \\
=\frac{4.3597 \times 10^{-18} \times 2.3071 \times 10^{-28} \times(137.036)^{2}}{\pi \times 1.6021 \times 10^{-19}}=3.7526 \times 10^{-23}(\mathrm{~J}) \\
\text { Error }=\frac{3.7526 \times 10^{-23}}{3.7629 \times 10^{-23}}-1=-0.00274
\end{gathered}
$$

\subsection{Ratio between the Gravitational Force and Electric Force}

Below, we present the empirical equation for the ratio between the gravitational force and electric force:

$$
\frac{G m_{p}^{2}}{\frac{e^{2}}{4 \pi \varepsilon_{0}}}=4.5 \times \frac{m_{e}}{e} \times \hbar c \times\left(1 \frac{\mathrm{C}}{\mathrm{J} \cdot \mathrm{m}} \times \frac{1}{1 \mathrm{~kg}}\right)
$$

where $1 \mathrm{~kg}$ is the standard unit of mass, as previously explained [1].

$$
\begin{gathered}
\frac{G m_{p}^{2}}{\frac{e^{2}}{4 \pi \varepsilon_{0}}}=\frac{6.6743 \times 10^{-11} \times\left(1.6726 \times 10^{-27}\right)^{2}}{2.3071 \times 10^{-28}}=8.0936 \times 10^{-37} \\
\hbar c=1.0546 \times 10^{-34} \times 2.9979 \times 10^{8}=3.1615 \times 10^{-26}(\mathrm{~J} \cdot \mathrm{m}) \\
4.5 \frac{m_{e}}{e} \hbar c=\frac{4.5 \times 9.1094 \times 10^{-31} \times 3.1615 \times 10^{-26}}{1.6021 \times 10^{-19}}=8.0889 \times 10^{-37} \\
\text { Error }=\frac{8.0936 \times 10^{-37}}{8.0889 \times 10^{-37}}-1=0.000578
\end{gathered}
$$

It is important to note that this error is small compared to the errors of the other empirical equations. The very large ratio between the gravitational force and electric force has long been a mystery in science. Such a simple empirical equation with high numerical accuracy has not been previously reported. Therefore, these values are emphasized here.

\section{Discussion}

\subsection{Relationships among Various Equations}

The relationships among several sets of equations are obvious. In this section, we explain these obvious relationships.

\subsubsection{Relationship among the Equations for the Four Special Lengths} Equations (9), (12), (15) and (18) indicate that

$$
r_{e} 2 \pi=\alpha \lambda_{e}=\alpha^{2} a_{0} 2 \pi=\alpha^{3} \frac{1}{2 R_{\infty}}
$$

Equation (33) is already known. From Equation (9),

$$
\frac{e^{3}}{r_{e} \pi\left(4 \pi \varepsilon_{0}\right)^{2}} \times\left(1 \frac{\mathrm{C}}{\mathrm{J} \cdot \mathrm{m}}\right)=k T_{c}
$$


$r_{e}$ can be defined as follows:

$$
m_{e} c^{2}=\frac{e^{2}}{4 \pi \varepsilon_{0} r_{e}}
$$

Then, the Coulomb's law with distance can be expressed in terms of the CMB as

$$
\frac{e^{2}}{4 \pi \varepsilon_{0} r^{2}}=\frac{1}{r^{2}} \frac{e \pi}{m_{e} c^{2}} k T_{c} \times\left(1 \frac{\mathrm{J} \cdot \mathrm{m}}{\mathrm{C}}\right)
$$

where $r$ is the distance between two electrons and $1 \mathrm{Jm} / \mathrm{C}$ is the standard electrostatic quantity, which is explained in a later section.

\subsubsection{Relationship among Equations (21), (25) and (36)}

From Equations (36),

$$
\frac{m_{e} c^{2}}{e \pi} \times \frac{e^{2}}{4 \pi \varepsilon_{0}}=k T_{c} \times\left(1 \frac{\mathrm{J} \cdot \mathrm{m}}{\mathrm{C}}\right)
$$

Equation (37) is equal to Equation (21). Next, the following equation is well known:

$$
E_{h}=\alpha^{2} m_{e} c^{2}
$$

Using Equations (37) and (38), we obtain

$$
E_{h} \times \frac{1}{\alpha^{2}} \times \frac{1}{e \pi} \times \frac{e^{2}}{4 \pi \varepsilon_{0}} \times\left(1 \frac{\mathrm{C}}{\mathrm{J} \cdot \mathrm{m}}\right)=k T_{c}
$$

Equation (39) is equal to Equation 25.

\subsection{Explanation of the Standard Electrostatic Quantity $(1 \mathrm{C} / \mathrm{J} / \mathrm{m})$}

As shown above, we can calculate the Coulomb potential energy with distance in terms of the CMB. Equation (34) is not complex. The difficulty in Equation (34) lies in the dimensional mismatch. Thus, we apply the majority of our efforts to resolving this dimensional mismatch.

$$
\begin{gathered}
\frac{e^{2}}{4 \pi \varepsilon_{0}}=2.3071 \times 10^{-28}(\mathrm{~J} \cdot \mathrm{m}) \\
n=\frac{1 \mathrm{C}}{e}=6.2415 \times 10^{18}
\end{gathered}
$$

where $n$ is the number of electrons.

$$
\frac{e^{2}}{4 \pi \varepsilon_{0}} \times n=\frac{e}{4 \pi \varepsilon_{0}} \times 1 \mathrm{C}=1.4400 \times 10^{-9}(\mathrm{~J} \cdot \mathrm{m})
$$

Therefore,

$$
\frac{e}{4 \pi \varepsilon_{0}}=1.4400 \times 10^{-9}\left(\frac{\mathrm{J} \cdot \mathrm{m}}{\mathrm{C}}\right)
$$

From Equation (5),

$$
\frac{e^{2}}{r_{e} \pi 4 \pi \varepsilon_{0}} \times \frac{e}{4 \pi \varepsilon_{0}} \times\left(1 \frac{\mathrm{C}}{\mathrm{J} \cdot \mathrm{m}}\right)=k T_{c}
$$


From Equations (43) and (44),

$$
\frac{e^{2}}{4 \pi \varepsilon_{0} r_{e} \pi} \times 1.4400 \times 10^{-9}=k T_{c}
$$

In Equation (45), the standard electrostatic quantity $(1 \mathrm{C} / \mathrm{J} / \mathrm{m})$ is absent, and $1.4400 \times 10^{-9}$ is a dimensionless constant. We have multiplied and divided by the number of electrons.

In Equation (42), $n$ is meaningful. However, in Equation (45), $n$ has disappeared. The right-hand side of Equation (44) is defined for only one electron. However, when $1 \mathrm{C}$ was defined as the standard charge, the relation to the number of electrons was unknown. If $10 \mathrm{C}$ were to be defined as the standard charge, then the number of electrons on the left-hand side would become ten times larger. In this case, the dimensionless constant $\left(1.4400 \times 10^{-9}\right)$ should be changed to $1.4400 \times 10^{-10}$ because of the division by the number of electrons. However, Equation (44) cannot be changed.

\subsection{Four Empirical Equations for Important Lengths without Using $\alpha$}

From Equation (33), it is unclear that $\lambda_{e}, r_{e}, a_{0}$, and $1 / 2 R_{\infty}$ are special lengths. In Equations (9), (12), (15) and (18), we have used $\alpha$. The definitions of $\alpha$ are as follows:

$$
\begin{gathered}
\frac{e^{2}}{4 \pi \varepsilon_{0} \hbar c}=\alpha=\frac{1}{137.036} \\
\frac{q_{m}^{2}}{\mu_{0} \pi \hbar c}=\frac{1}{\alpha}=137.036
\end{gathered}
$$

Thus,

$$
\frac{1}{\alpha}=\left(\frac{e^{2}}{4 \pi \varepsilon_{0} \hbar c}\right)^{-\frac{1}{2}}\left(\frac{q_{m}^{2}}{\mu_{0} \pi \hbar c}\right)^{\frac{1}{2}}=\left(\frac{e^{2}}{4 \pi \varepsilon_{0}}\right)^{-\frac{1}{2}}\left(\frac{q_{m}^{2}}{\mu_{0} \pi}\right)^{\frac{1}{2}}
$$

Using Equation (48), $\alpha$ can be eliminated from Equations (9), (12), (15) and (18). The error does not change.

$$
\begin{gathered}
\frac{1}{e \times \frac{2 r_{e} \pi}{2}} \times\left(\frac{e^{2}}{4 \pi \varepsilon_{0}}\right)^{2} \times\left(1 \frac{\mathrm{C}}{\mathrm{J} \cdot \mathrm{m}}\right)=k T_{c} \\
\frac{1}{e \times \frac{\lambda_{e}}{2}} \times\left(\frac{e^{2}}{4 \pi \varepsilon_{0}}\right)^{\frac{3}{2}} \times\left(\frac{q_{m}^{2}}{\mu_{0} \pi}\right)^{\frac{1}{2}} \times\left(1 \frac{\mathrm{C}}{\mathrm{J} \cdot \mathrm{m}}\right)=k T_{c} \\
\frac{1}{2 \pi a_{0}} \times\left(\frac{e^{2}}{4 \pi \varepsilon_{0}}\right)^{1} \times\left(\frac{q_{m}^{2}}{\mu_{0} \pi}\right)^{1} \times\left(1 \frac{\mathrm{C}}{\mathrm{J} \cdot \mathrm{m}}\right)=k T_{c} \\
\frac{1}{e \times \frac{1}{4 R_{\infty}}} \times\left(\frac{e^{2}}{4 \pi \varepsilon_{0}}\right)^{\frac{1}{2}} \times\left(\frac{q_{m}^{2}}{\mu_{0} \pi}\right)^{\frac{3}{2}} \times\left(1 \frac{\mathrm{C}}{\mathrm{J} \cdot \mathrm{m}}\right)=k T_{c}
\end{gathered}
$$


Equations (49), (50), (51) and (52) clearly show that $\lambda_{e}, r_{e}, a_{0}$, and $1 / 2 R_{\infty}$ are special lengths. We predict that $0.9937 \mu \mathrm{m}\left(=a_{0} \times 137.036 \times 137.036\right)$ is the fundamental special length. At this length, there should be an influence from the force between magnets near electrons. These special lengths have long been a mystery in science. Such simple empirical equations with high numerical accuracy have not been previously reported. Therefore, these values are emphasized here.

\subsection{Our Empirical Equations Are Not Coincidence}

We have presented many empirical equations. There are obvious relationships among several of these empirical equations, which clearly are not independent. However, the following three equations do seem to be independent.

Based on Equation (1), the gravitational force can be explained. For convenience, Equation (1) is rewritten below:

$$
\frac{G m_{p}}{\frac{\lambda_{p}}{2}} \times 1 \mathrm{~kg}=\frac{9}{2} k T_{c}
$$

Based on Equation (28), the ratio between the gravitational force and electric force can be explained. For convenience, Equation (28) is rewritten below:

$$
\frac{G m_{p}^{2}}{\frac{e^{2}}{4 \pi \varepsilon_{0}}}=4.5 \times \frac{m_{e}}{e} \times \hbar c \times\left(1 \frac{\mathrm{C}}{\mathrm{J} \cdot \mathrm{m}} \times \frac{1}{1 \mathrm{~kg}}\right)
$$

Based on Equation (36), the Coulomb's law with distance in terms of the CMB can be explained. For convenience, Equation (36) is rewritten below:

$$
\frac{e^{2}}{4 \pi \varepsilon_{0} r^{2}}=\frac{1}{r^{2}} \frac{e \pi}{m_{e} c^{2}} k T_{c} \times\left(1 \frac{\mathrm{J} \cdot \mathrm{m}}{\mathrm{C}}\right)
$$

The roles of these three empirical equations are clearly different.

We discovered Equation (53) first. Next, we discovered Equations (54) and (55). After the discovery of Equations (54) and (55), we noted that Equation (55) can be deduced from Equations (53) and (54). The mathematical proof is shown in Appendix A. We strongly believe that the mathematical connection among these three equations provides evidence that they are not coincidence.

\subsection{Comparison of Equation (1) and Equation (21)}

We will attempt to explain the various quantities considered dimensions and also to point out the major results in a more transparent way in this section.

The factor of 9/2 in Equation (1) can be explained as follows [2]. The proton consists of three quarks. Therefore, we must consider $9 / 2 k T$ and not $3 k T$. Accordingly, the number of degrees of freedom inside the proton may be 9 . From Equation (21),

$$
m_{e} c^{2} \times \frac{e}{4 \pi \varepsilon_{0}} \times\left(1 \frac{\mathrm{C}}{\mathrm{J} \cdot \mathrm{m}}\right)=\pi k T_{c}
$$


Based on our consideration to resolve the dimension mismatch problem in section 4.2, from Equation (56), the number of degrees of freedom inside the electron seems to be $2 \pi$. Much previous work has been done to unify different theories within a single equation/result. In particular, the recent work by Angrick et al. has done this for electronic structure calculations in quantum mechanics [5]. These authors showed that the spin of electrons cannot be ignored thermodynamically. Then, considering the spin, the number of degrees of freedom inside an electron may be different from 3. Furthermore, Aquino et al. discovered new methods using vector analysis [6]. Perhaps it can be inferred that there is an unknown relationship in the present electromagnetic vector analysis.

\section{Conclusions}

Previously, we discovered an empirical equation (Equation (1)) relating the gravitational force and the CMB. However, according to Jarzynski, Jarzynski's equality can be used to determine the electromagnetic force in quantum physics [3]. Therefore, we searched for further empirical equations. Thus, equations for the Coulomb's law with distance in terms of the CMB (Equation (36)) and the ratio between the gravitational force and electric force (Equation (28)) were discovered. Empirical equations for the Rydberg constant, Bohr radius, Compton wavelength and classical electron radius in terms of the CMB have also been presented here, along with their verification and errors. These lengths can be understood as special lengths related to the force between magnets. We predict that $0.9937 \mu \mathrm{m}$ should be the fundamental special length. These special lengths have long been a mystery in science. Such simple empirical equations with high numerical accuracy have not been previously reported. Regarding the ratio between the gravitational force and electric force, the very large ratio between these forces also has long been a mystery in science, and such a simple empirical equation with high numerical accuracy has not been previously reported. Unfortunately, the background theory still is not sufficiently complete. Therefore, we have abandoned the attempt to present any theoretical explanation in this report. We expect to publish such theoretical explanations in a future report.

Furthermore, we can show a clear connection among three different empirical equations. Thus, we conclude that our empirical equations are not coincidence.

\section{Conflicts of Interest}

The author declares no conflicts of interest regarding the publication of this paper.

\section{References}

[1] Miyashita, T. (2020) Journal of Modern Physics, 11, 1180-1192. https://doi.org/10.4236/jmp.2020.118074

[2] Miyashita, T. (2020) Journal of Modern Physics, 11, 1559-1560. https://doi.org/10.4236/jmp.2020.1110096 
[3] Jarzynski, C. (2017) Physical Review X, 7, Article ID: 011008. https://doi.org/10.1103/PhysRevX.7.011008

[4] Jacobson, T. (1995) Physical Review Letters, 75, 1260-1263. https://doi.org/10.1103/PhysRevLett.75.1260

[5] Angrick, C., Braun, J., Ebert, H. and Donath, M. (2021) Journal of Physics: Condensed Matter, 33, Article ID: 115501. https://doi.org/10.1088/1361-648X/abd338

[6] Aquino, F.W. and Wong, B.M. (2018) Journal of Physical Chemistry Letters, 9, 6456-6462. https://doi.org/10.1021/acs.jpclett.8b02786 


\section{Appendix A}

The mathematical connection of the three equations is shown in this appendix.

$$
\begin{gathered}
\frac{e^{2}}{4 \pi \varepsilon_{0} \hbar c}=\alpha=\frac{1}{137.036} \\
\frac{q_{m}^{2}}{\mu_{0} \pi \hbar c}=\frac{1}{\alpha}=137.036
\end{gathered}
$$

So,

$$
\hbar c=\left(\frac{e^{2}}{4 \pi \varepsilon_{0}}\right)^{\frac{1}{2}}\left(\frac{q_{m}^{2}}{\mu_{0} \pi}\right)^{\frac{1}{2}}
$$

For convenience, Equation (53) is rewritten as (A4).

$$
\frac{G m_{p}}{\frac{\lambda_{p}}{2}}=\frac{9}{2} k T_{c}
$$

Here,

$$
\lambda_{p}=\frac{h}{m_{p} c}
$$

From Equations (A4) and (A5),

$$
\frac{G m_{p} m_{p} c^{2}}{h c}=\frac{9}{4} k T_{c}
$$

From Equations (A3) and (A6),

$$
G m_{p}^{2}=\frac{4.5 \pi}{c^{2}}\left(\frac{e^{2}}{4 \pi \varepsilon_{0}}\right)^{\frac{1}{2}}\left(\frac{q_{m}^{2}}{\mu_{0} \pi}\right)^{\frac{1}{2}} k T_{c}
$$

For convenience, Equation (54) is rewritten as (A8).

$$
\frac{G m_{p}^{2}}{\frac{e^{2}}{4 \pi \varepsilon_{0}}}=4.5 \times \frac{m_{e}}{e} \times \hbar c
$$

From Equations (A3) and (A8),

$$
G m_{p}^{2}=4.5 \times \frac{m_{e}}{e} \times\left(\frac{e^{2}}{4 \pi \varepsilon_{0}}\right)^{\frac{3}{2}}\left(\frac{q_{m}^{2}}{\mu_{0} \pi}\right)^{\frac{1}{2}}
$$

For convenience, Equation (55) is rewritten as Equation (A10).

$$
\frac{m_{e} c^{2}}{e \pi} \times \frac{e^{2}}{4 \pi \varepsilon_{0}}=k T_{c}
$$

From Equations (A7) and (A9),

$$
\frac{4.5 \pi}{c^{2}}\left(\frac{e^{2}}{4 \pi \varepsilon_{0}}\right)^{\frac{1}{2}}\left(\frac{q_{m}^{2}}{\mu_{0} \pi}\right)^{\frac{1}{2}} k T_{c}=4.5 \frac{m_{e}}{e}\left(\frac{e^{2}}{4 \pi \varepsilon_{0}}\right)^{\frac{3}{2}}\left(\frac{q_{m}^{2}}{\mu_{0} \pi}\right)^{\frac{1}{2}}
$$

So, 


$$
\frac{\pi}{c^{2}} k T_{c}=\frac{m_{e}}{e} \times \frac{e^{2}}{4 \pi \varepsilon_{0}}
$$

So,

$$
\frac{m_{e} c^{2}}{e \pi} \times \frac{e^{2}}{4 \pi \varepsilon_{0}}=k T_{c}
$$

Equation (A13) is the same as Equation (A10).

Consequently, Equation (55) can be deduced from Equations (53) and (54). 